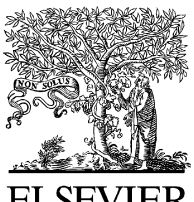

\title{
Complete screening for glucocerebrosidase mutations in Parkinson disease patients from Portugal
}

\author{
Jose Bras $^{\mathrm{a}, \mathrm{b}}$, Coro Paisan-Ruiz ${ }^{\mathrm{a}}$, Rita Guerreiro ${ }^{\mathrm{a}, \mathrm{b}}$, Maria Helena Ribeiro ${ }^{\mathrm{b}}$, Ana Morgadinho ${ }^{\mathrm{c}}$, \\ Cristina Januario $^{c}$, Ellen Sidransky ${ }^{\mathrm{d}}$, Catarina Oliveira $^{\mathrm{b}}$, Andrew Singleton ${ }^{\mathrm{a}, *}$ \\ ${ }^{a}$ Laboratory of Neurogenetics, National Institutes on Aging, National Institutes of Health, Bethesda, MD, USA \\ ${ }^{\mathrm{b}}$ Center for Neurosciences and Cell Biology, Faculty of Medicine, University of Coimbra, Coimbra, Portugal \\ ${ }^{c}$ Neurology Service, Coimbra University Hospital, Coimbra, Portugal \\ d Medical Genetics Branch, National Human Genome Research Institute, National Institutes of Health, Bethesda, MD, USA
}

Received 31 October 2007; accepted 14 November 2007

\begin{abstract}
Mutations in the gene encoding beta-glucocerebrosidase, a lysosomal degrading enzyme, have recently been associated with the development of Parkinson disease.

Here we report the results found in a cohort of Portuguese Parkinson disease patients and healthy age-matched controls for mutations in the aforementioned gene. This screening was accomplished by sequencing the complete open-reading frame, as well as intron/exon boundaries, of the glucocerebrosidase gene, in a total of 230 patients and 430 controls.

We have found an increased number of Parkinson disease patients presenting mutations in GBA when compared to controls.

These results, together with recent literature, clearly suggest a role of glucocerebrosidase in the development of Parkinson disease. (C) 2007 Elsevier Inc. All rights reserved.
\end{abstract}

Keywords: Case control study; Genetic association; Parkinson disease

\section{Introduction}

Mutations and complex rearrangements in the gene encoding $\beta$-glucocerebrosidase (GBA) cause Gaucher disease (GD), an autosomal recessive lysosomal storage disorder often categorized in three main sub-types: nonneuronopathic type 1 , acute neuronopathic type 2 and subacute neuronopathic type 3 . The clinical features in patients with GD vary greatly, ranging from asymptomatic octogenarians to severely affected infants with hepatosplenomegaly, developmental regression and growth arrest. The symptoms encountered in GD do not typically overlap significantly with those seen in patients with Parkinson disease (PD). However,

\footnotetext{
* Corresponding author at: Laboratory of Neurogenetics, National Institutes on Aging, National Institutes of Health, Building 35, Room 1A1014, 35 Lincoln Drive, Bethesda, MD 20892, USA. Tel.: +1 3014516079 ; fax: +1 3014515466 .

E-mail address: singleta@mail.nih.gov (A. Singleton).
}

there have been reports describing rare patients with GD who develop manifestations of PD. This prompted researchers to explore the prevalence of mutations in GBA among subjects with PD; the startling association between GBA mutations and PD in brain bank samples (Lwin et al., 2004) led others to assess Jewish and non-Jewish populations (Aharon-Peretz et al., 2004; Ziegler et al., 2007). Several replication studies have been published with positive and negative associations; the relative rarity of mutations and the complexity of the genomic region where GBA is associated resulted in studies that were underpowered or were focused only on specific mutations.

\section{Materials and methods}

Here we report the results of complete sequence analysis of GBA in a series of 230 Portuguese patients with PD, collected sequentially at the Coimbra University Hospital in 
Table 1

Variants found

\begin{tabular}{|c|c|c|c|c|c|c|c|}
\hline \multicolumn{2}{|c|}{ Mutations } & \multicolumn{3}{|c|}{ PD patients } & \multicolumn{3}{|c|}{ Controls } \\
\hline Protein $^{\mathrm{a}}$ & Allele name ${ }^{\mathrm{b}}$ & $N$ & $\%$ of all cases & $\%$ of carriers & $N$ & $\%$ of all controls & $\%$ of carriers \\
\hline N409S & N370S & 5 & 2.2 & 35.7 & 3 & 0.7 & 100 \\
\hline $\mathrm{N} 435 \mathrm{~T}$ & N396T & 5 & 2.2 & 35.7 & 0 & - & - \\
\hline D448H & D409H & 1 & 0.4 & 7.1 & 0 & - & - \\
\hline L483P & L444P & 3 & 1.3 & 21.4 & 0 & - & - \\
\hline \multicolumn{8}{|c|}{ Polymorphic variants } \\
\hline K13R & $\mathrm{K}(-27) \mathrm{R}$ & 1 & 0.4 & 20 & 0 & - & - \\
\hline $\mathrm{R} 41 \mathrm{~L}^{\mathrm{c}}$ & $\mathrm{R} 2 \mathrm{~L}^{\mathrm{c}}$ & 0 & - & - & 1 & 0.2 & 9.1 \\
\hline E365K & E326K & 2 & 0.9 & 40 & 3 & 0.7 & 27.3 \\
\hline $\mathrm{T} 408 \mathrm{M}$ & $\mathrm{T} 369 \mathrm{M}$ & 2 & 0.9 & 40 & 5 & 1.2 & 45.5 \\
\hline $\mathrm{E} 427 \mathrm{~K}^{\mathrm{c}}$ & E388K $\mathrm{K}^{\mathrm{c}}$ & 0 & - & - & 2 & 0.5 & 18.2 \\
\hline
\end{tabular}

As described in the text, all variants were present in heterozygous state.

a Amino acid designations are based on the primary GBA translation product, including the 39-residue signal peptide.

b Common nomenclature attributed to mutations; does not include the 39-residue signal peptide.

c These represent previously unpublished mutations, therefore pathogenicity or functional effects are unknown.

Portugal and in 430 healthy age-matched controls. All subjects were Caucasian and of apparent Portuguese ancestry. Diagnosis was made in accordance with the UK Brain Bank criteria. Family history was considered positive if at least one family member presented with PD. The entire coding region and the exon/intron boundaries of GBA gene were sequenced in all subjects (details available upon request). To exclude false positives due to co-amplification of the pseudogene, all mutations were confirmed by sequencing bands excised from an agarose gel, following PCR amplification of a fresh DNA aliquot.

\section{Results}

The PD group yielded 14 carriers of previously described pathogenic GBA mutations (N370S, N396T, D409H and L444P), all heterozygous, while the control group yielded 3 N370S carriers, also heterozygotes (Table 1). Two variants, E326K and T369M, previously described as non-pathogenic polymorphisms, were identified in both patients and controls. In addition, 2 novel variants and one previously described variant of unknown significance were identified (p.K13R; p.R41L and p.E427K) (Rozenberg et al., 2006).

\section{Discussion}

We found a frequency of $6.1 \%$ (14/230) known pathogenic mutations in the PD series and $0.7 \%(3 / 430)$ in the control group. These results represent a significantly higher frequency of mutations in GBA in PD patients when compared to controls $(p<0.001 ; \mathrm{OR}=9.2 ; 95 \%$ CI 2.6-32.4). Of note, the control group shows no mutations associated with severe GD; while they exist in the PD group - 4/14 patients with L444P or D409H. If we consider the variants of unknown pathogenicity (p.K13R; p.R41L and p.E427K) as potentially causative, this association still remains $(p<0.001 ; \mathrm{OR}=4.9$; 95\% CI 1.9-12.9).
The most common mutation identified was N370S, the most frequently identified pathogenic mutation in Ashkenazi Jewish as well as Portuguese patients with GD. Based on screening of 2000 random cord blood samples in Portugal, the carrier frequency of this mutation is estimated to be 0.0043 in this population (Lacerda et al., 1994). This mutation is believed to account for $63 \%$ of the mutant alleles in Portuguese patients with GD. Although the N370S mutation was three times more frequent in the PD group when compared to controls, we did not have sufficient power to identify a statistically significant association analyzing this mutation alone $(p=0.079$; OR 3.3; 95\% CI 0.75-13.4). Mutation N396T, encountered in 5 subjects in this study, was first identified in Portugal and has proven to be a relatively common mutation in this population (Amaral et al., 2000).

This study substantiates the need to sequence GBA in non-Ashkenazi cohorts in order to accurately determine the frequency of mutations in this gene. Had we screened only for common Gaucher mutations, we would have missed $43 \%$ of the mutant alleles in this population.

The association of GBA mutations with PD in the Portuguese population is particularly interesting when it is noted that the mutation driving this association is one associated with Jewish ancestry, and that another PD causing mutation, p.G2019S of LRRK2, underlying $\sim 6 \%$ of Portuguese PD cases (Bras et al., 2005), is also associated with Ashkenazi Jewish ancestry; these data clearly illustrate the contribution of Jewish ancestry to the modern Portuguese population.

In summary using a cohort with a different and defined ethnicity, we demonstrate that GBA mutations are significantly more common in patients with PD than in neurologically normal controls. These findings illustrate that the identification of such an association requires large sample series, even when using populations where GBA mutations are enriched; thus detection of such an association in populations with non-Ashkenazi ancestry is likely to require thousands of samples. 


\section{Conflict of interest statement}

None.

\section{Acknowledgements}

This work was supported in part by the Intramural program of the National Institute on Aging, National Institutes of Health, Department of Health and Human Services and Fundação para a Ciência e Tecnologia grants (SFRH/BD/ 29647/2006) and (SFRH/BD/27442/2006).

\section{References}

Aharon-Peretz, J., Rosenbaum, H., Gershoni-Baruch, R., 2004. Mutations in the glucocerebrosidase gene and Parkinson's disease in Ashkenazi Jews. N. Engl. J. Med. 351 (19), 1972-1977.
Amaral, O., Marcao, A., Sa Miranda, M., Desnick, R.J., Grace, M.E., 2000. Gaucher disease: expression and characterization of mild and severe acid beta-glucosidase mutations in Portuguese type 1 patients. Eur. J. Hum. Genet. 8 (2), 95-102.

Bras, J., Guerreiro, R., Ribeiro, M., Januário, C., Morgadinho, A., Oliveira, C., Cunha, L., Hardy, J., Singleton, A., 2005. G2019S Dardarin substitution is a common cause of Parkinson's disease in a Portuguese cohort. Movement Disord. 20 (12), 1653-1655.

Lacerda, L., Amaral, O., Pinto, R., Oliveira, P., Aerts, J., Sa Miranda, M.C., 1994. Gaucher disease: N370S glucocerebrosidase gene frequency in the Portuguese population. Clin. Genet. 45 (6), 298-300.

Lwin, A., Orvisky, E., Goker-Alpan, O., LaMarca, M.E., Sidransky, E., 2004. Glucocerebrosidase mutations in subjects with parkinsonism. Mol. Genet. Metab. 81 (1), 70-73.

Rozenberg, R., Fox, D.C., Sobreira, E., Pereira, L.V., 2006. Detection of 12 new mutations in Gaucher disease Brazilian patients. Blood Cells Mol. Dis. 37 (3), 204-209.

Ziegler, S.G., Eblan, M.J., Gutti, U., Hruska, K.S., Stubblefield, B.K., GokerAlpan, O., LaMarca, M.E., Sidransky, E., 2007. Glucocerebrosidase mutations in Chinese subjects from Taiwan with sporadic Parkinson disease. Mol. Genet. Metab. 91 (2), 195-200. 